\title{
PROJECTIVE UNIFORMIZATION, EXTREMAL CHERN CLASSES AND QUATERNIONIC SHIMURA CURVES
}

\author{
PRISKA JAHNKE AND IVO RADLOFF
}

\begin{abstract}
Projective structures on compact real manifolds are classical objects in real differential geometry. Complex manifolds with a holomorphic projective structure on the other hand form a special class as soon as the dimension is greater than one. In the Kähler Einstein case $\mathbb{P}_{m}$, tori and ball quotients are essentially the only examples. They can be described purely in terms of Chern class conditions. We give a complete classification of all projective manifolds carrying a projective structure. The only additional examples are modular abelian families over quaternionic Shimura curves. They can also be described purely in terms of Chern class conditions.
\end{abstract}

\section{INTRODUCTION}

The uniformization theorem of compact Riemann surfaces $M$ says the universal covering space $\tilde{M}$ admits an equivariant embedding into $\mathbb{P}_{1}(\mathbb{C})$, i.e., we may view $\tilde{M}$ as a submanifold of $\mathbb{P}_{1}(\mathbb{C})$ such that $\pi_{1}(M)$ becomes a subgroup of $\operatorname{PSl}_{1}(\mathbb{C})$. In higher dimensions this is no longer true. Manifolds $M_{m}, m>1$, with projective uniformization, i.e., the analogous property of $\tilde{M}_{m}$ admitting an equivariant embedding into $\mathbb{P}_{m}(\mathbb{C})$, form a very special class first studied by Kobayashi and Ochiai.

One of their results is the following generalization of the case of compact Riemann surfaces to higher dimensions ([KoOc80]):

0.1. Theorem. On a compact Kähler Einstein manifold $M$, the following conditions are equivalent:

1.) $M$ carries a holomorphic normal projective connection.

2.) The Chen Ogiue inequality $2(m+1) c_{2}(M) \leq m c_{1}^{2}(M)$ is an equality.

3.) $M$ is either $\mathbb{P}_{m}(\mathbb{C})$, a finite étale quotient of a torus or a ball quotient.

In any of these cases, $M$ admits a flat holomorphic normal projective connection.

A holomorphic normal projective connection is an infinitesimal version of projective uniformization $\tilde{M}_{m} \hookrightarrow \mathbb{P}(\mathbb{C})_{m}$. A ball quotient means $\tilde{M}_{m} \simeq \mathbb{B}_{m}(\mathbb{C})=\left\{z \in \mathbb{C}^{m}|| z \mid<1\right\}$. Note that if we identify $\mathbb{B}_{m}(\mathbb{C}) \subset \mathbb{C}^{m} \subset \mathbb{P}_{m}(\mathbb{C})$ in a standard way, then every affine transformation of $\mathbb{C}^{m}$ and in fact every automorphism of $\mathbb{B}_{m}(\mathbb{C})$ is induced by a projective transformation of $\mathrm{PSl}_{m}(\mathbb{C})$. This explains why the manifolds in 3.) are examples.

Kobayashi and Ochiai's result immediately raises the question of what are the non Kähler-Einstein manifolds $M_{m}$ with projective uniformization. The assumption is analytic in nature and therefore particularly interesting for algebraic projective $M_{m}$, i.e., manifolds that can be described as common zero set of a number of homogeneous algebraic equations in some projective space $\mathbb{P}_{N}(\mathbb{C})$.

Another result of Kobayashi and Ochiai says there are no examples in the surface case $([\mathrm{KoOc} 80])$ : a projective surface with projective uniformization is Kähler Einstein. Before the authors' previous work ([JaRa04a $]$ ) it was in fact unknown whether there exist any non-Kähler Einstein examples at all. In [JaRa04a the complete classification in the

Date: September 3, 2018.

1991 Mathematics Subject Classification. 53B10; 14D06; $14 \mathrm{~K} 10$. 
projective threefold case was given and it was proved that there exists exactly one source of non Kähler Einstein examples in three dimensions: modular families of false elliptic curves over a Shimura curve (see (0.3)).

In this article the classification is extended to arbitrary dimensions in the flat case:

0.2. Theorem. On a projective manifold $M_{m}$ which is $\underline{\text { not }}$ Kähler Einstein, the following conditions are equivalent:

1.) $M$ carries a flat holomorphic normal projective connection.

2.) Up to a finite étale covering, $M$ admits an abelian group scheme structure $f$ : $M \longrightarrow C$ over a compact Shimura curve $C$ such that the Arakelov inequality $2 \operatorname{deg} f_{*} \Omega_{M / C}^{1} \leq(m-1) \operatorname{deg} K_{C}=(m-1)\left(2 g_{C}-2\right)$ ([Fa83]) is an equality.

3.) Up to a finite étale covering, $M \simeq Z \times_{C} Z \times_{C} \cdots \times_{C} Z$, where $Z \longrightarrow C$ is a Kuga fiber space constructed from the rational corestriction $\operatorname{Cor}_{F / \mathbb{Q}}(A)$ of a division quaternion algebra $A$ defined over a totally real number field $F$ such that

$$
A \otimes_{\mathbb{Q}} \mathbb{R} \simeq M_{2}(\mathbb{R}) \oplus \mathbb{H} \oplus \cdots \oplus \mathbb{H} .
$$

Here $\mathbb{H}$ denotes the Hamiltonian quaternions (see section $[5$ ).

Consequently, if $M_{m}$ is a projective manifold whose universal covering space can be embedded equivariantly into $\mathbb{P}_{m}(\mathbb{C})$, then $M_{m}$ is either Kähler Einstein as in (0.1) or a modular abelian fibration over a Riemann surface as in (0.2) (and $\tilde{M} \simeq \mathbb{C}^{m-1} \times \mathfrak{H}_{1}$ ).

By a result of Viehweg and Zuo, 2.) implies 3.) ([ViZu04]). The construction of the family $Z \longrightarrow C$ in 3.) is a generalization of a construction of modular families of abelian varieties due to Mumford (Mu66]). The curve $C$ can be embedded as a totally geodesic rigid submanifold of some moduli space of abelian varieties.

Even though the examples are well known, they have apparently never before been considered as examples of manifolds carrying a projective structure. From the point of view of hermitian symmetric spaces, these additional examples have to do with $I_{1,1} \simeq I I I_{1}$, i.e., the one dimensional ball is a Siegel space. The flatness assumption in 1.) is necessary for some geometric arguments in section 3 .

For the proof of (0.2) see sections 4 and 5. The construction of the examples in 3.) is explained in detail in section 5 . We only give some details here and the simplest case in (0.3). The rational corestriction $\operatorname{Cor}_{F / \mathbb{Q}}(A)$ is a central simple $\mathbb{Q}$-algebra of dimension $4^{d}$, where $d:=[F: \mathbb{Q}]$, and either

$$
\operatorname{Cor}_{F / \mathbb{Q}}(A) \simeq M_{2^{d}}(\mathbb{Q}) \quad \text { or } \quad \operatorname{Cor}_{F / \mathbb{Q}}(A) \simeq M_{2^{d-1}}(B)
$$

for some division quaternion algebra $B / \mathbb{Q}$. We refer to the first case as ' $B$ splits'. From this setting one constructs ([Mu66], [ViZu04]) a modular family $f: Z \longrightarrow C$ of abelian varieties over some compact Shimura curve $C$ with simple general fiber $Z_{\tau}$ satisfying

- $\operatorname{dim} Z_{\tau}=2^{d-1}$ and $E n d_{\mathbb{Q}}\left(Z_{\tau}\right) \simeq \mathbb{Q}$ in the case $B$ split,

- $\operatorname{dim} Z_{\tau}=2^{d}$ and $E n d_{\mathbb{Q}}\left(Z_{\tau}\right) \simeq B$ in the case $B$ non-split.

The smallest possible dimension is obtained for $F=\mathbb{Q}, A=B$ an indefinite division quaternion algebra ( $B$ is necessarily non-split). Here $f: Z \longrightarrow C$ is a (PEL-type) family of abelian surfaces, $\operatorname{End}_{\mathbb{Q}}\left(Z_{\tau}\right) \simeq B$ for $\tau$ general. Such abelian surfaces are called false elliptic curves, it is the additional three dimensional example found in [JaRa04a].

The construction of this family is recalled in 2.) below. Also included in 1.) is the case of modular families of elliptic curves. These are non-compact examples and of no furhter interest for our purposes, but they explain the general idea and show why such families arise in this context.

0.3. Example. 1.) Modular families of elliptic curves. Let $\Gamma \subset S l_{2}(\mathbb{Z})$ be torsion free, for example some congruence subgroup. Then $\Gamma$ acts without fixed points on $\mathfrak{H}_{1}$ as a group of 
moebius transformations, $C=\Gamma \backslash \mathfrak{H}_{1}$ is a Riemann surface with projective uniformization by construction. It is not possible to choose $\Gamma$ such that $\Gamma \backslash \mathfrak{H}_{1}$ is compact.

Let $\Lambda=\mathbb{Z} \oplus \mathbb{Z}$ and denote by $\Gamma_{\Lambda} \simeq \Lambda \rtimes \Gamma$ the group of matrices

$$
\left(\begin{array}{ccc}
1 & m & n \\
0 & a & b \\
0 & c & d
\end{array}\right) \subset S l_{3}(\mathbb{R}), \quad(m, n) \in \Lambda, \quad \gamma=\left(\begin{array}{ll}
a & b \\
c & d
\end{array}\right) \in \Gamma
$$

Think of $\mathbb{C} \times \mathfrak{H}_{1}$ as an open set of $\mathbb{P}_{2}$ by identifying $(z, \tau)$ and $[z: \tau: 1]$. The group $\Gamma_{\Lambda}$ acts on $\mathbb{C} \times \mathfrak{H}_{1}$ projectively by

$$
(z, \tau) \mapsto\left(\frac{z+m \tau+n}{c \tau+d}, \frac{a \tau+b}{c \tau+d}\right),
$$

again without fixed points. The quotient $S=\Gamma_{\Lambda} \backslash \mathbb{C} \times \mathfrak{H}_{1}$ is a smooth quasi projective surface with projective uniformization by construction. It comes with a smooth and proper elliptic fibration $f: S \longrightarrow C=\Gamma \backslash \mathfrak{H}_{1}$, the fiber over $[\tau]$ is the elliptic curve $\simeq \mathbb{C} /(\mathbb{Z} \tau+\mathbb{Z})$.

However, $S$ will non-compact and every smooth compactification will add rational curves to $S$ in the new fibers and this will destroy the projective uniformization (3.3).

2.) Modular families of false elliptic curves ([Sh59]). A false elliptic curve is an abelian surface $A$ whose $\mathbb{Q}$-Endormorphismring is a totally indefinite quaternion algebra over $\mathbb{Q}$. Modular families of such surfaces can be constructed as follows.

Start with a totally indefinite quaternion algebra $B$ over $\mathbb{Q}$, for example the algebra $\subset M_{2 \times 2}(\mathbb{R})$ generated over $\mathbb{Q}$ by

$$
x=\left(\begin{array}{cc}
\sqrt{2} & 0 \\
0 & -\sqrt{2}
\end{array}\right), \quad y=\left(\begin{array}{cc}
0 & -3 \\
1 & 0
\end{array}\right)
$$

Then $x^{2}=2, y^{2}=-3$ (omitting $1_{2 \times 2}$ ), $x y=-y x$ and $B=\mathbb{Q}+\mathbb{Q} x+\mathbb{Q} x+\mathbb{Q} x y$. Let $\Lambda \simeq \mathbb{Z}^{\oplus 4}$ be some maximal order of $B$, for example (see [KoVe03]

$$
\Lambda=\mathbb{Z}+\frac{1}{2} \mathbb{Z}(x+x y)+\frac{1}{2} \mathbb{Z}(1+y)+\mathbb{Z} x y .
$$

Let $\Gamma$ be a torsion free subgroup of $\Lambda_{1}^{\times}$, the norm one unit subgroup of $\Lambda$. Then $\Gamma \subset \mathrm{Sl}_{2}(\mathbb{R})$ acts without fixed points on $\mathfrak{H}_{1}$. Contrary to the case of elliptic curves, $\Gamma$ may be chosen such that $C=\Gamma \backslash \mathfrak{H}_{1}$ is compact.

Denote by $\Gamma_{\Lambda} \simeq \Lambda \rtimes \Gamma$ the group of matrices

$$
\left(\begin{array}{ll}
1_{2 \times 2} & \lambda \\
0_{2 \times 2} & \gamma
\end{array}\right) \subset S l_{4}(\mathbb{R}), \quad \lambda \in \Lambda, \quad \gamma \in \Gamma
$$

Think of $\mathbb{C}^{2} \times \mathfrak{H}_{1}$ as an open subset of $\mathbb{P}_{3}$ by identifying $(z, \tau)$ and $[z: \tau: 1], z=\left(z_{1}, z_{2}\right)$. As in the case of elliptic curves, the group $\Gamma_{\Lambda}$ acts on $\mathbb{C}^{2} \times \mathfrak{H}_{1}$ projectively. The quotient $T=\Gamma_{\Lambda} \backslash \mathbb{C}^{2} \times \mathfrak{H}_{1}$ is a smooth compact threefold whose projective uniformization directly follows from the construction. It comes with a smooth and proper false elliptic curve fibration $f: T \longrightarrow C$, the fiber over $[\tau]$ is $A_{\tau} \simeq \mathbb{C}^{2} / \Gamma\left(\begin{array}{l}\tau \\ 1\end{array}\right)$. For $\tau$ general End $\mathbb{Q}_{\mathbb{Q}}\left(A_{\tau}\right) \simeq B$. In fact $T$ is projective (5.18).

We should mention that there is a notion of an $S$-structure for an arbitrary hermitian symmetric space $S$ of the compact type. It is a useful tool for example in connection with rigidity questions ([KoOc81], [HwMo98]). The rank one case $S=\mathbb{P}_{m}(\mathbb{C})$ we consider here is somewhat special in this context.

Acknowledgements. The authors want to thank N. Nakayama for valuable explanations concerning abelian fibrations as well as E. Viehweg and K. Zuo for explanations concerning their work. 
Notations. We consider complex Kähler manifolds $M$, maps are holomorphic or rational. $T_{M}$ denotes the holomorphic tangent bundle, $\Omega_{M}^{1}$ the dual bundle of holomorphic one forms. We do not distinguish between line bundles and divisors on $M . K_{M}=\operatorname{det} \Omega_{M}^{1}$ denotes the canonical divisor.

\section{Holomorphic NORMAL PROJECTIVE CONNECTIONS}

Following classical notation ([Gu78], [KoOc80]) we study manifolds with a holomorphic projective structure or connection. We assume $M$ compact Kähler, $m=\operatorname{dim} M$.

1.1. Holomorphic projective structures. Let $V \simeq \mathbb{C}^{m+1}$ and $\mathbb{P}_{m}=\mathbb{P}(V)$. The group $S l_{m+1}(\mathbb{C})$ acts on $\mathbb{P}_{m}$ with kernel $Z\left(S l_{m+1}(\mathbb{C})\right)=\mu_{m+1}=\left\{\lambda \cdot I d_{m+1} \mid \lambda^{m+1}=1\right\}$. We obtain a finite covering map of degree $m+1$

$$
\mathrm{Sl}_{m+1}(\mathbb{C}) \longrightarrow \operatorname{Aut}(\mathbb{P}(V))=\mathrm{PSl}_{m}(\mathbb{C}) .
$$

In fact $\mathrm{Sl}_{m+1}(\mathbb{C})$ is the universal covering space of $\operatorname{PSl}_{m}(\mathbb{C})$. For later use note that $Z\left(S l_{m+1}(\mathbb{C})\right)$ is also the kernel of the $m+1$-th symmetric power map.

1.2. Definition. $M$ admits a holomorphic projective structure, if there exists an atlas $\left\{\left(U_{i}, \varphi_{i}\right)\right\}_{i \in I}$ with holomorphic maps $\varphi_{i}: U_{i} \hookrightarrow \mathbb{P}_{m}(\mathbb{C})$ such that

$$
\varphi_{i} \circ \varphi_{j}^{-1}: \varphi_{j}\left(U_{i j}\right) \longrightarrow \varphi_{i}\left(U_{i j}\right)
$$

is the restriction of some $g_{i j} \in \operatorname{PSl}_{m}(\mathbb{C})$ whenever $U_{i j}=U_{i} \cap U_{j} \neq \emptyset$.

We will say $M$ admits a projective structure for short. The $g_{i j}$ define a class $\xi \in$ $H^{1}\left(M, \mathrm{PSl}_{m}(\mathbb{C})\right)$. Let $G_{i j} \in S l_{m+1}(\mathbb{C})$ be a set of matrices representing $g_{i j}$. The $G_{i j}$ do not necessarily define a class in $H^{1}\left(M, S l_{m+1}(\mathbb{C})\right)$ as $\xi$ does not necessarily lift in

$$
H^{1}\left(M, S l_{m+1}(\mathbb{C})\right) \longrightarrow H^{1}\left(M, \operatorname{PSl}_{m}(\mathbb{C})\right) \longrightarrow H^{2}\left(M, \mu_{m+1}\right) .
$$

The obstruction space $H^{2}\left(M, \mu_{m+1}\right)$ is non empty for $M$ projective. An manifold with projective uniformization as in the introduction clearly admits a projective strucutre.

1.3. Example. 1.) Kähler examples include $\mathbb{P}_{m}(\mathbb{C})$, finite étale quotients of tory, ball quotients (see the introduction). Note that this is the list of Kähler manifolds of constant holomorphic sectional curvature. Note also that $\mathbb{B}_{m}(\mathbb{C})$ is the non compact dual of $\mathbb{P}_{m}(\mathbb{C})$ in the sense of hermitian symmetric spaces.

2.) We find more examples if we drop the assumption $M$ projective/Kähler: certain Hopf manifolds or twistor spaces over conformally flat Riemannian fourfolds.

3.) If $M$ carries a projective structure, then so does every étale covering of $M$.

1.4. Development. (KoOc80, $[$ KoWu83] $)$ Let $M$ be as in (1.2). Denote by $\tilde{M}$ the universal covering space of $M$. Fixing a starting point $p \in \tilde{M}$, we find an immersive $\operatorname{map} \psi: \tilde{M} \rightarrow \mathbb{P}_{m}(\mathbb{C})$ and a map $\rho: \pi_{1}(M, \pi(p)) \simeq \pi_{1}(M) \rightarrow P G l_{m}(\mathbb{C})$ such that $\psi(\gamma(p))=\rho(\gamma)(\psi(p))$ for any $\gamma \in \pi_{1}(M)$. The map $\psi$ is called a development. It is obtained as follows:

A point on $\tilde{M}$ corresponds to a pair $[q, \sigma]$ where $q \in M$ and $\sigma$ is a path from $\pi(p)$ to $q$ in $M$. Cover the trace of $\sigma$ by open subsets $\left(U_{i}, \varphi_{i}\right), i=1, \ldots, r$, as in (1.2), starting at $\pi(p) \in U_{1}$ and ending at $q \in U_{r}$. Put $\psi([q, \sigma])=\left(g_{1,2} \circ \cdots \circ g_{r-1, r}\right)\left(\varphi_{1}(\pi(p))\right.$. In the case $q=\pi(p)$ one has $\sigma=: \gamma \in \pi_{1}(M, \pi(p))$ and one defines $\rho(\gamma)=\left(g_{1,2} \circ \cdots \circ g_{r-1, r}\right)$.

If conversely the universal covering space $\tilde{M}$ of some complex manifold $M$ admits an immersive equivariant map $\tilde{M} \longrightarrow \mathbb{P}_{m}(\mathbb{C})$, then $M$ carries a projective structure.

1.5. Example. If $M$ is simply connected and $M$ carries a projective structure, then $M \simeq \mathbb{P}_{m}(\mathbb{C})$. 
1.6. Induced bundles. Let $M$ be as in 1.2 . Let $E$ be a holomorphic $\operatorname{PSl}_{m}(\mathbb{C})-$ homogeneous vector bundle on $\mathbb{P}(V)$, i.e., a vector bundle with the property that the action of $\mathrm{PSl}_{m}(\mathbb{C})$ on $\mathbb{P}(V)$ extends to the total space $E$. Then $E$ induces a holomorphic vector bundle on $M$. Indeed, the local pieces $\varphi_{i}^{*} E$ defined on $U_{i} \subset M$ glue.

This is not clear for $\mathrm{Sl}_{m+1}(\mathbb{C})$-homogeneous bundles, i.e bundles where the action of $\mathrm{Sl}_{m+1}(\mathbb{C})$ on $\mathbb{P}(V)$ extends. As a prototype example consider

$$
\emptyset_{\mathbb{P}(V)}(1)=-\frac{K_{\mathbb{P}(V)}}{m+1}
$$

This line bundle is $S l_{m+1}(\mathbb{C})$ homogeneous but not $\mathrm{PSl}_{m}(\mathbb{C})$ homogeneous. In other words, $\emptyset_{\mathbb{P}(V)}(1)$ comes from the representation of the parabolic subgroup of $\mathrm{Sl}_{m+1}(\mathbb{C})$

$$
P=\left\{\left(\begin{array}{cc}
A & 0 \\
b^{t} & a
\end{array}\right) \mid A \in G l_{m}(\mathbb{C}), b \in \mathbb{C}^{m}, a \in \mathbb{C}^{*}, \operatorname{det} A \cdot a=1\right\}
$$

given by projection onto $a$. But this representation is not induced by a representation of $\mathbb{P}(P) \subset \mathrm{PSl}_{m}(\mathbb{C})$.

If $E$ is a $S l_{m+1}(\mathbb{C})$-homogeneous bundle and if the representation of $P$ commutes with $S^{m+1}$, then $S^{m+1} E$ is a $\mathrm{PSl}_{m}(\mathbb{C})$ homogeneous bundle. It is the case in the above example: $S^{m+1} \emptyset_{\mathbb{P}(V)}(1) \simeq K_{\mathbb{P}(V)}$ is $\mathrm{PSl}_{m}(\mathbb{C})$ homogeneous, the induced bundle on $M$ is $-K_{M}$. The obstruction of extracting the $m+1$-th root of $K_{M}$ in $\operatorname{Pic}(M)$ again lies in $H^{2}\left(M, \mu_{m+1}\right)$.

1.8. Lemma. On every manifold $M$ with a projective structure we have a surjection

$$
B \longrightarrow-K_{M} \longrightarrow 0
$$

where $B$ is some rank $r>0$ flat vector bundle coming from a representation $\pi_{1}(M) \longrightarrow$ $S l_{r}(\mathbb{C})$.

Proof. In (1.7), projection onto $A$ or $a$ gives two standard representations of $P$. As a $P$ module, $V$ sits in an extension $0 \longrightarrow K \longrightarrow V \longrightarrow L \longrightarrow 0$ where $K$ and $L$ are complex vector spaces of dimension $\operatorname{dim} K=m, \operatorname{dim} L=1$. The induced sequence of $\mathrm{Sl}_{m+1}(\mathbb{C})$ homogeneous bundles on $\mathbb{P}(V)$ is, up to dualizing and twist, the Euler sequence or first jet sequence

$$
0 \longrightarrow \Omega_{\mathbb{P}(V)}^{1} \otimes \varnothing_{\mathbb{P}(V)}(1) \longrightarrow V \otimes \varnothing_{\mathbb{P}(V)} \longrightarrow \varnothing_{\mathbb{P}(V)}(1) \longrightarrow 0 .
$$

The $m+1$-th symmetric power gives a surjection of homogeneous $\operatorname{PSl}_{m}(\mathbb{C})$ bundles $S^{m+1}\left(V \otimes \emptyset_{\mathbb{P}(V)}\right) \longrightarrow-K_{\mathbb{P}(V)}=\varnothing_{\mathbb{P}(V)}(m+1)$. On $M$ we obtain a surjection $B \longrightarrow-K_{M}$ where $B$ is flat. Of course $B$ is just the bundle induced by $S^{m+1} G_{i j}$ for a choice of $G_{i j}$ as in (1.2)

There is an infinitesimal description of projective structures:

1.11. Holomorphic normal projective connections. Recall the definition of the Atiyah class ([At57]): Associated to a holomorphic vector bundle $E$ on the complex manifold $M$ one has the first jet sequence

$$
0 \longrightarrow \Omega_{M}^{1} \otimes E \longrightarrow J_{1}(E) \longrightarrow E \longrightarrow 0 .
$$

The obstruction to the holomorphic splitting is a class $b(E) \in H^{1}\left(M, H o m(E, E) \otimes \Omega_{M}^{1}\right)$ called Atiyah class of $E$. For properties of $b(E)$ see [At57]. We only mention that if $\Theta^{1,1}$ denotes the $(1,1)$-part of the curvature tensor of some differentiable connection on $E$, then, under the Dolbeault isomorphism, $b(E)$ corresponds to $\left[\Theta^{1,1}\right] \in H^{1,1}(M, \operatorname{Hom}(E, E))$. In particular, as $M$ is Kähler, $\operatorname{tr}(b(E))=-2 i \pi c_{1}(E) \in H^{1}\left(M, \Omega_{M}^{1}\right)$. This is why we normalise and put $a(E):=-\frac{1}{2 i \pi} b(E)$. 
1.12. Definition. $M_{m}$ carries a holomorphic normal projective connection if the (normalised) Atiyah class of the holomorphic cotangent bundle has the form

$$
a\left(\Omega_{M}^{1}\right)=\frac{c_{1}\left(K_{M}\right)}{m+1} \otimes i d_{\Omega_{M}^{1}}+i d_{\Omega_{M}^{1}} \otimes \frac{c_{1}\left(K_{M}\right)}{m+1} \in H^{1}\left(M, \Omega_{M}^{1} \otimes T_{M} \otimes \Omega_{M}^{1}\right),
$$

where we use $\Omega_{M}^{1} \otimes T_{M} \otimes \Omega_{M}^{1} \simeq \Omega_{M}^{1} \otimes \operatorname{End}\left(\Omega_{M}^{1}\right) \simeq \operatorname{End}\left(\Omega_{M}^{1}\right) \otimes \Omega_{M}^{1}$.

We will say $M$ has a projective connection for short. It was shown in MoMo96 how a holomorphic cocycle solution to (1.13) can be thought of as a $\mathbb{C}$-bilinear holomorphic connection map $\Pi: T_{M} \times T_{M} \rightarrow T_{M}$ satisfying certain rules modelled after the Schwarzian derivative. Conversely, the existence of such a connection implies (1.13). We will not use this description.

1.14. Proposition. If $M$ admits a projective structure, then $M$ admits a projective connection. The connection is called flat in this case.

For a proof see [KoOc80]. It is not known whether all projective manifolds admitting a projective connection also admit a flat projective connection. $M$ admits a projective structure if and only if $\Pi=0$ is a cocycle solution to (1.13). $M$ carries a flat projective connection iff $M$ admits a projective structure.

1.15. Chern Classes. Let $M_{m}$ be as above compact Kähler with a projective connection. As in the case of projectice space one has ([KoOc80]):

$$
c_{r}(M)=\frac{1}{(m+1)^{r}}\left(\begin{array}{c}
m+1 \\
r
\end{array}\right) c_{1}^{r}(M), r=0, \ldots, m \quad \text { in } H^{r}\left(M, \Omega_{M}^{r}\right) .
$$

In particular, $2(m+1) c_{2}(M)=m c_{1}^{2}(M)$.

\section{KäHLER Einstein MANifOLDS}

In the Kähler Einstein case one has (0.1) Theorem from the introduction. We include the proof for the convenience of the reader: The Chen-Ogiue inequality (ChOg75) says that on $\left(M_{m}, \omega\right)$ compact Kähler-Einstein

$$
\int_{M}\left(m c_{1}^{2}(M)-2(m+1) c_{2}(M)\right) \wedge \omega^{m-2} \leq 0,
$$

with equality if and only if $(M, \omega)$ is of constant holomorphic sectional curvature. A compact complex manifold $M_{m}$ carries a Kähler metric of constant holomorphic sectional curvature $s$ if and only if

- $M \simeq \mathbb{P}_{m}(\mathbb{C})$ (case $s>0$ ) or

- $M$ is an étale quotient of a torus (case $s=0$ ) or

- $M$ is a ball quotient (case $s<0$ ).

This is the equivalence of 2.) and 3.) in (0.1) Theorem. In the case of 1.) we have (1.16), implying equality in (2.1). Hence 1.) implies 2.). By example 1.3 the third point 3.) implies 1.). The proof of (0.1) is complete.

\section{Projective Manifolds}

Let $M_{m}$ be a projective manifold with a projective connection, not necessarily flat. Recall that a line bundle $L$ is called nef, if $L . C \geq 0$ for every irreducible curve $C$ in $M$. A vector bundle $E$ is nef, if $\varnothing_{\mathbb{P}(E)}(1)$ is nef. A nef line bundle is big if $L^{m}>0$.

The manifold is called minimal if $K_{M}$ is nef. It is called a good minimal model, if $\left|d K_{M}\right|$ is spanned for $d \gg 0$. In this case one has the Iitaka fibration

$$
f: M \longrightarrow Y \text {. }
$$


Here $Y$ is normal, fibers are connected. One of the central conjectures in birational algebraic geometry predicts: $M$ minimal implies $M$ is a good minimal model. The conjecture is proven in dimension $\leq 3$ but open in higher dimension.

3.2. Rational Curves. We say that $M$ contains a rational curve, if there exists a non constant holomorphic map $\nu: \mathbb{P}_{1}(\mathbb{C}) \longrightarrow M$.

3.3. Proposition. Let $M_{m}$ be a projective manifold with a projective connection. If $M$ contains a rational curve, then $M \simeq \mathbb{P}_{m}(\mathbb{C})$.

We include here a proof of the flat case, for the general case see JaRa04a.

Proof. Let $\psi: \tilde{M} \longrightarrow \mathbb{P}_{m}(\mathbb{C})$ be a development of the universal covering space $\mu: \tilde{M} \longrightarrow$ $M$. We have an induced map $\tilde{\nu}: \mathbb{P}_{1}(\mathbb{C}) \longrightarrow \tilde{M}$ such that $\mu \circ \tilde{\nu}=\nu$. Then $\nu^{*} T_{M}=$ $(\psi \circ \tilde{\nu})^{*} T_{\mathbb{P}_{m}(\mathbb{C})}$ is ample. This forces $M \simeq \mathbb{P}_{m}(\mathbb{C})$ by Mori's proof of Hartshorne's conjecture ([Mori79], in particular [MiPe97] I, Theorem 4.2.).

3.4. Remark. 1.) The assumption $M$ projective (Kähler) is necessary as soon as $\operatorname{dim} M>$ 2 ([KoOc80]). Indeed, the twistor space $T w(S)$ over a compact conformally flat real fourfold $S$ is a compact complex threefold with a projective structure. Any such $T w(S)$ is differentiably covered by rational curves. There are choices for $S$ such that $T w(S) \neq \mathbb{P}_{3}(\mathbb{C})$. But then $T w(S)$ is not Kähler ([Hi81]).

2.) (3.3) holds mutatis mutandis for any projective manifold $M$ with a flat $S$-structure, $S$ an irreducible hermitian symmetric space of the compact type: on any rational curve, $T_{M}$ is nef by the above argument, implying $M$ uniruled. In the case of $\operatorname{rank} S>1$ we have $M \simeq S$ by [HwMo97.

3.5. Corollary. Let $M_{m} \nsucceq \mathbb{P}_{m}(\mathbb{C})$ be a projective manifold with a projective connection. Then

1.) $K_{M}$ is nef and $K_{F}$ is nef for every smooth submanifold $F \subset M$.

2.) any rational map $M^{\prime}$......... $>M$ from a complex manifold $M^{\prime}$ must be holomorphic.

Proof. As $M_{m} \neq \mathbb{P}_{m}(\mathbb{C}), M_{m}$ is free of rational curve by (3.3). Then 1.) follows from the cone theorem [KoMo98].

2.) if we had to blow up $M^{\prime}$ in order to make the map holomorphic, then $M$ would contain a rational curve.

Combining (0.1) and (3.3) and deep results of Aubin and Yau we find:

3.6. Corollary. Let $M_{m}$ be a projective manifold with a projective connection

1.) if $K_{M}$ is big and nef, then $M$ is a ball quotient.

2.) if $K_{M} \equiv 0$, then $M$ is a finite étale quotient of a torus.

Proof. If $K_{M} \equiv 0$, then $M$ admits a Kähler-Einstein metric [Ya78]. We coclude by (0.1).

If $K_{M}$ is big and nef, then $\left|d K_{M}\right|$ is spanned for $d \gg 0$ by the base point free theorem KoMo98. The Iitaka fibration $f: M \longrightarrow Y$ is birational, exceptional fibers are covered by rational curves by [Ka91, Theorem 2. By (3.3) $M$ is free of rationale curves, so $f$ is an isomorphism. Then $K_{M}$ is ample. Then $M$ is Kähler-Einstein Au76]. We again conclude by (0.1).

3.7. Abelian group schemes. A fibration $f: M \longrightarrow Y$ is an abelian group scheme, if $Y$ is smooth, $f$ is submersive, every fiber of $f$ is a smooth abelian variety, and $f$ admits a smooth section. Our aim is to prove:

3.8. Theorem. Let $M_{m} \not \mathbb{P}_{m}(\mathbb{C})$ carry a projective structure. Then, up to a finite étale cover, $M$ is an abelian group scheme over a base $N$ of general type. 
The proof can be found at the end of this section. First note some special cases: the example of $M$ a finite étale quotient of an abelian variety is the case $\operatorname{dim} N=0$. The case of $M$ a ball quotient is contained in the case $\operatorname{dim} N=\operatorname{dim} M$.

The proof of (3.8) uses a result of Kollár. Recall from ( Ko93, 1.7.) that $M$ is said to have (generically) large fundamental group if for any irreducible complex subvariety $Z \subset M$ of positive dimension (passing through a general point)

$$
\operatorname{Im}\left[\pi_{1}\left(Z_{\text {norm }}\right) \longrightarrow \pi_{1}(M)\right] \quad \text { is infinite. }
$$

Here $Z_{\text {norm }}$ denotes the normalization of $Z$.

3.9. Proposition. Any $M \nsucceq \mathbb{P}_{m}(\mathbb{C})$ with a projective structure has large fundamental group.

Proof. By 3.5, $K_{M}$ is nef. We prove by contradiction. Assume $\operatorname{Im}\left[\pi_{1}\left(Z_{\text {norm }}\right) \rightarrow \pi_{1}(M)\right]$ is finite for some $Z$ as above. Denote the normalization map by $\nu: Z_{\text {norm }} \rightarrow Z$.

Let $C_{n o r m}$ be some general curve in $Z_{\text {norm }}$, i.e., the intersection of $\operatorname{dim} Z-1$ general hyperplane sections. Think of $C_{\text {norm }}$ as the normalization of $C:=\nu\left(C_{\text {norm }}\right) \subset Z$. We have

$$
\pi_{1}\left(C_{\text {norm }}\right) \longrightarrow \pi_{1}\left(Z_{\text {norm }}\right) \longrightarrow \pi_{1}(M) .
$$

Then $\operatorname{Im}\left[\pi_{1}\left(C_{\text {norm }}\right) \longrightarrow \pi_{1}(M)\right]$ is finite. The kernel of $\pi_{1}\left(C_{\text {norm }}\right) \longrightarrow \pi_{1}(M)$ induces a finite étale covering $C^{\prime} \longrightarrow C_{\text {norm }}$ from a compact Riemann surface $C^{\prime}$, such that $\mu: C^{\prime} \longrightarrow C \subset M$ factors over $\tilde{M}$, the universal covering space of $M$. Let $\psi: \tilde{M} \longrightarrow$ $\mathbb{P}_{m}(\mathbb{C})$ be a development. Denote the induced map $C^{\prime} \longrightarrow \tilde{M} \longrightarrow \mathbb{P}_{m}(\mathbb{C})$ by $\psi_{1}$. Then $\mu^{*} T_{M}=\psi_{1}^{*} T_{\mathbb{P}_{m}(\mathbb{C})}$ is ample, implying $-K_{M} . C>0$. This contradicts $K_{M}$ nef.

3.10. Proposition. For any $M \not \mathbb{P}_{m}(\mathbb{C})$ with a projective structure there exists a finite étale cover $M^{\prime} \longrightarrow M$ such that $M^{\prime}$ is a good minimal model (see (3.1)) and the Iitaka fibration $f: M^{\prime} \longrightarrow Y$ satisfies

1.) $Y$ is birational to a smooth $W$ of general type.

2.) a general fiber of $f$ is a finite étale quotient of an abelian variety.

Proof. By 3.5. $K_{M}$ is nef. We have to prove $\left|d K_{M}\right|$ is spanned for $d \gg 0$. We may replace $M$ by finite étale covers. By 1.8 we have a surjection

$$
B \longrightarrow-K_{M} \longrightarrow 0
$$

from a flat bundle $B$ of some rank $r$ which is induced by a representation

$$
\rho: \pi_{1}(M) \longrightarrow \mathrm{Sl}_{r}(\mathbb{C})
$$

For a holomorphic $h: Z \longrightarrow M$, we have the map $\pi_{1}(Z) \longrightarrow \pi_{1}(M)$ and composition gives a representation of $\pi_{1}(Z)$. We call it the "pull back of $\rho$ to $Z$ ". It will be denoted $h^{*} \rho$.

1. Case. $\rho$ has a finite image. Then there exists a finite étale covering $\nu: \tilde{M} \longrightarrow M$ such that $\nu^{*} B$ is trivial. The pull back of (3.11) shows that $-K_{\tilde{M}} \simeq \nu^{*}\left(-K_{M}\right)$ is globally generated. On the other hand $K_{\tilde{M}}$ is nef. Then $K_{\tilde{M}} \simeq \varnothing_{\tilde{M}}$.

2. Case. $\rho$ has an infinite image. Denote by $G$ the component of the identity of the Zariski closure of $\rho\left(\pi_{1}(M)\right)$. After a finite étale cover we may assume $\rho\left(\pi_{1}(M)\right) \subset G$. Let $\operatorname{Rad}(G)$ be the solvable radical of $G$.

Assume $G$ is not solvable. Then $G / \operatorname{Rad}(G)$ is semisimple. Consider $\bar{\rho}: \pi_{1}(M) \longrightarrow$ $G / \operatorname{Rad}(G)$ obtained by composition. The image is Zariski dense. By [Ko93], 3.5., there exists $g: M \cdots \cdots \cdots \cdots$ dominant to some smooth $W$ of dimension $\operatorname{dim} W>0$ with the following property: if $Z \subset M$ passes through a very general point, then $Z$ will be contracted by $g$ if and only if

$$
\bar{\rho}\left(\operatorname{Im}\left(\pi_{1}\left(Z^{\text {norm }}\right) \longrightarrow \pi_{1}(M)\right)\right) \subset G / \operatorname{Rad}(G)
$$


is finite. Here $W$ is a smooth model of $\operatorname{Sh}_{k e r n \bar{\rho}}(M)$. We denote by $\pi: M_{1} \longrightarrow M$ a resolution such that $g_{1}: M_{1} \longrightarrow W$ is holomorphic.

After replacing $M$ by a finite étale cover we may instead of finiteness assume $\pi^{*} \bar{\rho}=g_{1}^{*} \sigma$ for some $\operatorname{big} \sigma: \pi_{1}(W) \longrightarrow G / \operatorname{Rad}(G)$ ([Zu96], explanations after Theorem 1). We claim that $W$ is of general type. In the case $G / \operatorname{Rad}(G)$ almost simple this is [Zu96], Theorem 1. The general case easily follows by induction on the number of almost simple almost direct factors of $G / \operatorname{Rad}(G)$ and $C_{n, m}$ from [Ka85.

The map $g$ is almost holomorphic by [Ko93], 4.1., i.e., there exist Zariski open dense subsets $M^{0} \subset M$ and $W^{0} \subset W$ such that $M^{0} \longrightarrow W^{0}$ is proper. Denote a general proper fiber of $g$ by $F$.

\subsection{Lemma. $F$ is an étale quotient of an abelian variety.}

The Lemma also applies to the case $G=\operatorname{Rad}(G)$ solvable. It proves in this case that $M$ is an étale quotient of an abelian variety.

Proof of (3.12). Denote the inclusion map by $\iota: F \hookrightarrow M$. The pull back $\iota^{*} \bar{\rho}$ is trivial so $\iota^{*} \rho: \pi_{1}(F) \longrightarrow \operatorname{Rad}(G)$. We find a basis of our representation space such that $\rho\left(\iota_{*} \gamma\right)$ has upper triangular form for any $\gamma \in \pi_{1}(F)$. This gives a filtration

$$
0 \subset E_{1} \subset E_{2} \subset \cdots \subset E_{r}=\iota^{*} B
$$

into flat bundles $E_{i}$ of rank $i$ on $F$. Each quotient $E_{i+1} / E_{i}$ is a flat line bundle on $F$, i.e., is induced by a representation of $\pi_{1}(F)$.

Flat line bundles are nef (this is not true for vector bundles of $r k>1$ ). Extensions of nef bundles are nef. This shows $\iota^{*} B$ is nef. Quotients of nef vector bundles bundles are nef. The pull back of (3.11) shows $\iota^{*}\left(-K_{M}\right)$ is nef. By adjunction, using the fact that $g$ is almost holomorphic, $\iota^{*}\left(-K_{M}\right) \simeq-K_{F}$. On the other hand $K_{F}$ is nef by (3.5). This shows $K_{F} \equiv 0$.

By the Bogomolov-Beauville decomposition theorem ([Bo74], Be83]) there exists an étale covering

$$
A \times Z \longrightarrow F
$$

where $A$ is abelian and $Z$ simply connected. By (3.9),$\pi_{1}(Z)=\{i d\}$ implies $Z$ is a point. Therefore, $F$ is covered by an abelian variety. This completes the proof of (3.12).

We continue the proof of (3.10). By [Ka85], $C_{n, m}$ is true for $g_{1}$, so

$$
\kappa(M)=\kappa\left(M_{1}\right) \geq \kappa(F)+\kappa(W)=\operatorname{dim} W .
$$

Hence, if $f: M \cdots \cdots \cdots$ denotes the rational Iitaka fibration, then $\operatorname{dim} Y=\kappa(M) \geq$ $\operatorname{dim} W$. On the other hand (3.12) implies that $f$ contracts the general fiber of $g$. This gives a rational dominant map $W \ldots \ldots \ldots \ldots . \rightarrow Y$. Then $Y$ and $W$ are birational.

By [Lai1], 4.4., $M$ has a good minimal model. By [Lai11], 2.5., $M$ is a good minimal model, as $K_{M}$ is nef. This completes the proof of (3.10).

Proof of (3.8). Replace $M$ by the finite étale cover from (3.10), denote by $f: M \longrightarrow Y$ the Iitaka fibration whose general fibers are finite étale quotients of abelian varieties. As $M$ is not Kähler Einstein, $0<\operatorname{dim} Y<\operatorname{dim} M$ by (3.6).

By (3.9), $M$ has large fundamental group. By [Ko93], 6.3. Theorem, some finite étale cover of $M$ is birational to an abelian group scheme $\alpha: A \longrightarrow S$. Replace $M$ by this cover. We arrived at the diagram

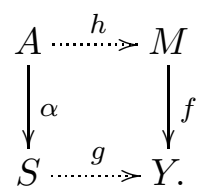


By (3.5), $h$ is holomorphic. The map $\alpha$ has a smooth section $s$. Then $g=f \circ h \circ s: S \longrightarrow Y$ must be holomorphic, too. By Ka91, Theorem 2, $f$ is equidimensional. Let $F$ be some fiber of $f$ over $q \in Y$ with induced scheme structure. The map $h$ is finite, generically $1: 1$ on every fiber $A_{p}$ of $\alpha$. Diagram (3.13) shows $F$ is irreducible and reduced (consider local sections of $\alpha)$. For $p \in g^{-1}(q)$ we may think of $h_{p}=\left.h\right|_{A_{p}}: A_{p} \longrightarrow F_{r e d}$ as the normalization of $F$.

Let $Y^{\prime}=h(s(S))$. Any positive dimensional fiber of $g: S \longrightarrow Y$ is covered by some fiber of $h: A \longrightarrow M$. Fibers of a birational morphism between smooth manifolds are rationally chain connected. As $M$ does not contain any rational curve, $h \circ s$ must contract every fiber of $S \longrightarrow Y$ to a point. Then $Y^{\prime} \simeq Y$. Now $Y^{\prime} . F=1$ implies $Y^{\prime} \simeq Y$ smooth. By (3.10), $Y$ is of general type.

Since $Y$ and $M$ are both smooth and $f$ is flat, the relative dualizing sheaf $\omega_{M / Y}=$ $\omega_{M} \otimes f^{*} \omega_{Y}^{-1}$ is locally free, hence every fiber $F$ of $f$ is Gorenstein with trivial canonical bundle $K_{F} \simeq \mathcal{O}_{F}$. Then $K_{A_{p}}=h_{p}^{*} K_{F}+\mathcal{N}$ where $\mathcal{N}$ is the conductor ideal. As $K_{A_{p}}$ and $K_{F}$ are both trivial, $\mathcal{N}$ must be trivial. Then $F$ is normal and $h_{p}$ is an isomorphism. Then $M \longrightarrow N:=Y$ is an abelian group scheme. This completes the proof of (3.8).

3.14. Example. 1.) Let $\Gamma \subset S l_{2}(\mathbb{R})$ be torsion free such that $C=\Gamma \backslash \mathfrak{H}_{1}$ is a smooth Riemann surface. Then $a(\gamma, \tau):=c \tau+d$ is a well defined factor of automorphy on $\mathfrak{H}_{1}$. It defines a theta characteristic which we denote $\frac{K_{C}}{2}$. The standard representation $\rho: \Gamma \longrightarrow S l_{2}(\mathbb{R})$ induces a flat bundle $E$ on $C$. It comes with an extension

$$
0 \longrightarrow \frac{K_{C}}{2} \longrightarrow E \longrightarrow-\frac{K_{C}}{2} \longrightarrow 0,
$$

the first jet sequence of $-\frac{K_{C}}{2} . E$ is flat but not nef. Here $G=S l_{2}(\mathbb{C})$ is almost simple.

2.) Consider the elliptic curve example $S=\Gamma_{\Lambda} \backslash \mathbb{C} \times \mathfrak{H}_{1}$ from $(0.3), 1$.) with proper elliptic fibration $f: S \longrightarrow C$. Here $K_{S} \simeq 3 f^{*}\left(\frac{K_{C}}{2}\right)$ where $\frac{K_{C}}{2} \in \operatorname{Pic}(C)$ denotes the theta characteristic as in 1.).

The standard representation $\rho: \Gamma_{\Lambda} \longrightarrow \mathrm{Sl}_{3}(\mathbb{C})$ induces a flat bundle $E$ on $S$. It comes with an extension

$$
0 \longrightarrow \Omega_{S}^{1} \otimes f^{*}\left(-\frac{K_{C}}{2}\right) \longrightarrow E \longrightarrow f^{*}\left(-\frac{K_{C}}{2}\right) \longrightarrow 0 .
$$

It is the first jet sequence of $-\frac{K_{S}}{3}=f^{*}\left(-\frac{K_{C}}{2}\right)$ as in the proof of (1.8). Restricted to a fiber of $S \longrightarrow C, E$ is given by the pull back representation

$$
(m, n) \mapsto\left(\begin{array}{ccc}
1 & m & n \\
0 & 1 & 0 \\
0 & 0 & 1
\end{array}\right)
$$

and we obtain the filtration as in the proof of (3.12). If $F$ denotes the non-splitting extension of $\varnothing$ and $\varnothing$ on the elliptic curve, then $E$ is the non-splitting extension of $\varnothing$ and $F$. Using the notation from the proof of (3.10), $\operatorname{Rad}(G) \simeq\left(\mathbb{C}^{2},+\right)$ is non trivial while $G / \operatorname{Rad}(G) \simeq \mathrm{Sl}_{2}(\mathbb{C})$ is almost simple.

\section{Abelian group schemes}

Because of (3.8) we consider from now on an abelian group scheme $f: M_{m} \longrightarrow N_{n}$ where $M$ carries a holomorphic normal projective connection. We have the exact sequence

$$
0 \longrightarrow f^{*} \Omega_{N}^{1} \stackrel{d f}{\longrightarrow} \Omega_{M}^{1} \longrightarrow \Omega_{M / N}^{1} \longrightarrow 0
$$

of holomorphic forms and

$$
E=E^{1,0}=f_{*} \Omega_{M / N}^{1}
$$


is a holomorphic rank $m-n$ vector bundle on $N$ such that $f^{*} E \simeq \Omega_{M / N}^{1}$ via the canonical map $f^{*} f_{*} \Omega_{M / N}^{1} \rightarrow \Omega_{M / N}^{1}$. Our aim is to prove

4.2. Theorem. In the above situation, assume in addition that $M$ is not Kähler-Einstein. Then $N$ is a compact Riemann surface and

$$
2 \operatorname{deg} E^{1,0}=(m-1) \operatorname{deg}\left(K_{N}\right)
$$

i.e., the Arakelov inequality is an equality.

Recall the Arakelov inequality ([Fa83, [JoZu02]): if $f: M \longrightarrow N$ is an abelian group scheme over a compact Riemann surface $N$, then $2 \operatorname{deg} E^{1,0} \leq(m-1) \operatorname{deg}\left(K_{N}\right)$. The theorem will be proved at the end of this section.

4.3. Proposition. In the above general situation of an abelian group scheme (Kähler Einstein or not), $N$ admits a projective connection, and

$$
a\left(E\left(-\frac{K_{N}}{n+1}\right)\right)=0 \quad \text { in } H^{1}\left(N, \operatorname{End}(E) \otimes \Omega_{N}^{1}\right),
$$

where a denotes the normalised Atiyah class.

4.5. Remark. 1.) The formula is in terms of classes, we do not assume the existence of a theta characteristic on $N$, i.e., a line bundle $\frac{K_{N}}{n+1} \in \operatorname{Pic}(N)$.

2.) In the case $N$ a compact Riemann surface, (4.4) implies $E \simeq U \otimes \frac{K_{N}}{2}$ for some flat bundle $U$ coming from a representation of $\pi_{1}(N)$ ([At57]) and some theta characteristic $\frac{K_{N}}{2} \in \operatorname{Pic}(N)$.

Some consequences first. The trace of the (normalised) Atiyah class gives the first Chern class, hence

$$
c_{1}(E)=\frac{m-n}{n+1} c_{1}\left(K_{N}\right) \quad \text { in } H^{1}\left(N, \Omega_{N}^{1}\right) .
$$

Let as usual $K_{M / N}=K_{M}-f^{*} K_{N}$. Then $K_{M / N}=\operatorname{det} \Omega_{M / N}^{1}=f^{*} \operatorname{det} E$. We may rewrite (4.6) as follows:

4.7. Corollary. In the situation of 4.3) the following identities hold in $H^{1}\left(M, \Omega_{M}^{1}\right)$ :

$$
c_{1}\left(K_{M / N}\right)=\frac{m-n}{n+1} c_{1}\left(f^{*} K_{N}\right) \quad \text { and } \quad c_{1}\left(K_{M}\right)=\frac{m+1}{n+1} c_{1}\left(f^{*} K_{N}\right) .
$$

In particular, $c_{1}\left(K_{M}\right)$ and $c_{1}\left(f^{*} K_{N}\right)$ are proportional.

\subsection{Corollary.}

1.) If $K_{M} \equiv 0$ or $K_{N} \equiv 0$, then $M$ and $N$ are étale quotients of abelian varieties.

2.) If $K_{M}$ or $K_{N}$ are not nef, then $M \simeq N \simeq \mathbb{P}_{m}(\mathbb{C})$.

Proof. 1.) By (4.7), if $K_{M} \equiv 0$ or $K_{N} \equiv 0$, then $K_{M} \equiv 0$ and $K_{N} \equiv 0$. The claim follows from (3.6) and (4.3).

2.) If $K_{M}$ or $K_{N}$ are not nef, then $K_{M}$ and $K_{N}$ are not nef by (4.7). Then $M \simeq \mathbb{P}_{m}(\mathbb{C}$ ) and $N \simeq \mathbb{P}_{n}(\mathbb{C})$ by (3.3). As $n>0$ by assumption, $m=n$ and $f$ is an automorphism of projective space.

Proof of (4.3). Denote the section of $f$ by $s: N \rightarrow M$. Consider the pull back to $N$ by $s$ of (4.1):

$$
0 \longrightarrow \Omega_{N}^{1} \stackrel{s^{*} d f}{\longrightarrow} s^{*} \Omega_{M}^{1} \longrightarrow s^{*} \Omega_{M / N}^{1} \simeq E \longrightarrow 0 .
$$

We have the map $d s: s^{*} \Omega_{M}^{1} \longrightarrow \Omega_{N}^{1}$. As $(d s)\left(s^{*} d f\right)=d(f \circ s)=i d_{\Omega_{N}^{1}}$, (4.9) splits holomorphically. 
The normalised Atiyah class of $s^{*} \Omega_{M}^{1}$ as a bundle on $N$ is obtained from $a\left(\Omega_{M}^{1}\right)$ by applying $d s$ to the last $\Omega_{M}^{1}$ factor in (1.13). We obtain

$$
a\left(s^{*} \Omega_{M}^{1}\right)=\frac{s^{*} c_{1}\left(K_{M}\right)}{m+1} \otimes d s+i d_{s^{*} \Omega_{M}^{1}} \otimes \frac{c_{1}\left(s^{*} K_{M}\right)}{m+1} \text { in } H^{1}\left(N, s^{*} \Omega_{M}^{1} \otimes s^{*} T_{M} \otimes \Omega_{N}^{1}\right),
$$

where we carefully distinguish between $s^{*} c_{1}\left(K_{M}\right) \in H^{1}\left(N, s^{*} \Omega_{M}^{1}\right)$ and the class $c_{1}\left(s^{*} K_{M}\right)=$ $d s\left(s^{*} c_{1}\left(K_{M}\right)\right) \in H^{1}\left(N, \Omega_{N}^{1}\right)$.

The Atiyah class of a direct sum is the direct sum of the Atiyah classes ([At57]). As the pull back of (4.1) splits holomorphically, we get the Atiyah classes of $\Omega_{N}^{1}$ and $E$ by projecting (4.10) onto the corresponding summands.

We first compute $a(E)$. The class $c_{1}\left(K_{M}\right) \in H^{1}\left(M, \Omega_{M}^{1}\right)$ is the pull back of some class in $H^{1}\left(N, \Omega_{N}^{1}\right)$; it therefore vanishes under $H^{1}$ of $\Omega_{M}^{1} \rightarrow \Omega_{M / N}^{1}$. This means the first summand in (4.10) vanishes if we project, while the second summand becomes

$$
i d_{E} \otimes \frac{c_{1}\left(s^{*} K_{M}\right)}{m+1} \text { in } H^{1}\left(N, E \otimes E^{*} \otimes \Omega_{N}^{1}\right) .
$$

This is $a(E)$. The trace gives

$$
c_{1}(E)=r k(E) \frac{c_{1}\left(s^{*} K_{M}\right)}{m+1} \in H^{1}\left(N, \Omega_{N}^{1}\right) .
$$

The determinant of (4.9) combined with the last formula and $r k E=m-n$ gives

$$
c_{1}\left(K_{N}\right)=c_{1}\left(s^{*} K_{M}\right)-c_{1}(E)=\frac{n+1}{m+1} c_{1}\left(s^{*} K_{M}\right)
$$

in $H^{1}\left(N, \Omega_{N}^{1}\right)$. Now (4.4) follows from (4.11).

Next we compute $a\left(\Omega_{N}^{1}\right)$. We first have to apply $d s$ to the first factor of the first summand in (4.10). This gives $\frac{c_{1}\left(s^{*} K_{M}\right)}{m+1}$. Contracting the middle factor using $s^{*} d f$ we obtain

$$
\frac{c_{1}\left(s^{*} K_{M}\right)}{m+1} \otimes i d_{\Omega_{N}^{1}}+i d_{\Omega_{N}^{1}} \otimes \frac{c_{1}\left(s^{*} K_{M}\right)}{m+1} \in H^{1}\left(N, \Omega_{N}^{1} \otimes T_{N} \otimes \Omega_{N}^{1}\right) .
$$

This is $a\left(\Omega_{N}^{1}\right)$. After replacing $c_{1}\left(s^{*} K_{M}\right)$ with the formula found in (4.12), we see that $N$ admits a projective connection (compare (1.13) $)$. This comples the proof of (4.3).

Proof of (4.2). By (3.8), the Iitaka fibration $f: M \longrightarrow N$ is an abelian group scheme where $N$ is of general type. As $M$ is not Kähler Einstein, $0<\operatorname{dim} N<\operatorname{dim} M$ by (3.6). By (4.3),$N$ has a projective connection. By (3.6), $N$ is a ball quotient, so $K_{N}$ is ample.

For any torsion free sheaf $\mathcal{F}$ on $N$ of positive rank and ample $H \in \operatorname{Pic}(N)$ the $H$-slope is defined $\mu_{H}(\mathcal{F})=\frac{c_{1}(\mathcal{F}) \cdot H^{n-1}}{\mathrm{rk} \mathcal{F}}$ as usual. Let $\mathbb{V}:=R^{1} f_{*} \mathbb{C}$. By [ViZu07], Theorem 1 and Remark 2, we have $\mu_{K_{N}}(\mathbb{V})=2 \mu_{K_{N}}(E) \leq \mu_{K_{N}}\left(\Omega_{N}^{1}\right)$. By (4.7)

$$
2 \mu_{K_{N}}(E)=\frac{2}{n+1} c_{1}\left(K_{N}\right)^{n} \leq \mu_{K_{N}}\left(\Omega_{N}^{1}\right)=\frac{1}{n} c_{1}\left(K_{N}\right)^{n} .
$$

Since $c_{1}\left(K_{N}\right)^{n}>0$ we find $n=1$. Then $N$ is Riemann surface and we have in fact equality $2 \mu_{K_{N}}(E)=\mu_{K_{N}}\left(\Omega_{N}^{1}\right)$, i.e., the Arakelov inequality is an equality.

We summarize our results in the first part of the proof of the main theorem (0.2):

Proof of 1.) $\Rightarrow$ 2.) $\Rightarrow$ 3.) in (0.2). From (3.8) and (4.2) we obtain 1.) $\Rightarrow$ 2.). By [ViZu04, Theorem 0.5., 2.) $\Rightarrow 3$.).

It remains to show that the examples in 3.), (0.2) indeed carry a projective structure. Before we come to this we add some explanations for the convenience of the reader: 
4.14. Remark. Let $f: M \longrightarrow N$ be an abelian group scheme over a compact Rieman surface $N$ with a projective connection. The push forward of (4.1) gives the Kodaira Spencer map

$$
E=f_{*} \Omega_{M / N}^{1} \longrightarrow K_{N} \otimes R^{1} f_{*} \varnothing_{M} .
$$

It is an isomorphism in the present case ([ViZu04], Proposition 1.2.). As a consequence of Simpson's correspondence, $E$ is poly stable, i.e., a direct sum of stable bundles of the same slope ([ViZu04], Proposition 1.2.). Kobayashi Hitchin correspondence and (4.3) implies $E \simeq U \otimes \frac{K_{N}}{2}$ for some unitary flat bundle $U$ and some theta characteristic $\frac{K_{N}}{2} \in \operatorname{Pic}(N)$ on $N$ (compare remark 4.5). The isomorphism (4.15) gives $R^{1} f_{*} \varnothing_{M} \simeq U \otimes\left(-\frac{K_{N}}{2}\right)$.

There exists a lift of $\pi_{1}(N)$ to a torsion free subgroup $\Gamma \subset S l_{2}(\mathbb{R})$, s.t. $R^{1} f_{*} \mathbb{C}$ comes from the tensor product of the canonical representation of $\Gamma$ and some unitary representation ([ViZu04], Proposition 1.4., Lemma 4.1.). We may assume that the representation induces $U$. Then

$$
0 \longrightarrow E \longrightarrow R^{1} f_{*} \mathbb{C} \otimes \varnothing_{N} \longrightarrow R^{1} f_{*} \varnothing_{M} \longrightarrow 0
$$

is the unique non-splitting extension of $-\frac{K_{N}}{2}$ by $\frac{K_{N}}{2}$ tensorized by $U$. In fact one has a tensor product description of $R^{1} f_{*} \mathbb{Q}$ defined over a number field. That $\Gamma$ comes from a quaternion algebra as in 3.) of (0.2) is in the end a consequence of a result of Takeuchi ([Ta75]). The tensor description of $R^{1} f_{*} \mathbb{Q}$ will play a role in the next section.

\section{Projective NON-KäHLER-Einstein EXAMPles}

The aim of this section is to prove that the explicit examples in 3.) of (0.2) carry a flat projective structure. The examples are well known families of abelian varieties ([Sh59], [Mu66], ViZu04, vGe08]) and have been studied from many points of view, but it was apparently unknown that they carry such a structure.

5.1. Data. Let $A$ be a division quaternion algebra defined over some totally real number field $F$ of degree $[F: \mathbb{Q}]=d$. Assume that $A$ splits at exactly one infinite place, i.e.,

$$
A \otimes \mathbb{Q} \mathbb{R} \simeq M_{2}(\mathbb{R}) \oplus \mathbb{H} \oplus \cdots \oplus \mathbb{H} .
$$

The existence of such $A$ 's follows from Hilbert's reciprocity law. Let $\operatorname{Cor}_{F / \mathbb{Q}}(A)$ be the rational corestriction of $A$. Then

$$
\operatorname{Cor}_{F / \mathbb{Q}}(A)=M_{2^{d-1}}(B),
$$

where $B$ is a quaternionen algebra over $\mathbb{Q}$, possibly split $(\underline{5.12})$ ). From this data we construct in section 5.10

1.) a torsion free discrete subgroup $\Gamma$ of $S l_{2}(\mathbb{R})$ acting canonically on $U_{\mathbb{R}}=\mathbb{R}^{2}$ such that $\Gamma \backslash \mathfrak{H}_{1}$ is compact,

2.) an orthogonal representation $\rho: \Gamma \longrightarrow O(g)$ on $W_{\mathbb{R}} \simeq \mathbb{R}^{g}$ (where $g=2^{d-1}$ in the case $B$ split and $g=2^{d}$ in the case $B$ non split), such that

3.) the symplectic representation $i d \otimes \rho$ fixes some complete lattice in $U_{\mathbb{R}} \otimes W_{\mathbb{R}}$ on which the symplectic form only takes integral values.

We will first explain how the above data leads to an abelian group scheme $M_{\Gamma} \longrightarrow C_{\Gamma}=$ $\Gamma \backslash \mathfrak{H}_{1}$ with a projective structure. Here $M_{\Gamma}$ will be compact if and only if $C_{\Gamma}$ is which is the case if the above data is indeed derived from a division quaternion algebra. For an explicit example see (0.3).

5.3. Construction of the abelian scheme $M \longrightarrow C$. Assume first we have a collection of data 1.) - 3.). How to derive it from a quaternion algebra will be shown in section 5.10 , 
(i) Choice of bases. Point 1.) includes a choice of a basis of $U_{\mathbb{R}} \simeq \mathbb{R}^{2}$. For later use it is more appropriate to choose an arbitrary basis of $W_{\mathbb{R}}$, not necessarily orthogonal. Write the elements of $U_{\mathbb{R}}$ and $W_{\mathbb{R}}$ as vertical vectors in the chosen bases. The action of $\Gamma$ on $U_{\mathbb{R}}$ is by left multiplication. The standard symplectic form on $U_{\mathbb{R}}$

$$
\left\langle u, u^{\prime}\right\rangle=u^{t} J_{2} u^{\prime}, \quad J_{2}:=\left(\begin{array}{cc}
0 & 1 \\
-1 & 0
\end{array}\right)
$$

identifies $S l_{2}(\mathbb{R})=S p_{2}(\mathbb{R})$ and $U_{\mathbb{R}} \simeq U_{\mathbb{R}}^{*}$ as $\Gamma$-modules. Write the elements of $U_{\mathbb{R}}^{*}$ in the dual base as horizontal vectors. The action of $\Gamma$ is then given by right multiplication $\gamma(u)=u \gamma^{-1}$, the $\Gamma$-isomorphism $U_{\mathbb{R}} \simeq U_{\mathbb{R}}^{*}$ by $u \mapsto u^{t} J_{2}$.

The choice of a basis for $U_{\mathbb{R}}$ and $W_{\mathbb{R}}$ gives an isomorphism

$$
W_{\mathbb{R}} \otimes U_{\mathbb{R}}^{*} \simeq M_{g \times 2}(\mathbb{R}) .
$$

Fix this isomorphism and think of the elements of $W_{\mathbb{R}} \otimes U_{\mathbb{R}}^{*}$ as real $g \times 2$ matrices from now on. Any $\gamma \in \Gamma$ acts by

$$
\gamma(\alpha)=\rho(\gamma) \alpha \gamma^{-1}, \quad \alpha \in M_{g \times 2}(\mathbb{R}) .
$$

Since $W_{\mathbb{R}} \otimes U_{\mathbb{R}}^{*} \simeq W_{\mathbb{R}} \otimes U_{\mathbb{R}}$ as $\Gamma$-modules, we find a complete lattice $\Lambda \subset M_{g \times 2}(\mathbb{R})$ invariant under the action of $\Gamma$ by 3.). The points 2.) and 3.) say we find a $\rho(\Gamma)$ invariant symmetric and positiv definit $S \in M_{g}(\mathbb{R})$, s.t. the induced symplectic form on $M_{g \times 2}(\mathbb{R})$ given by

$$
E(\alpha, \beta):=\operatorname{tr}\left(\alpha^{t} S \beta J_{2}\right)
$$

takes only integral values on $\Lambda$. For later considerations note that $M_{g \times 2}(\mathbb{R}) \simeq W_{\mathbb{R}} \otimes U_{\mathbb{R}}^{*}$ is a symplectic $O(S) \times S l_{2}(\mathbb{R})$ module via $(\delta, \gamma)(\alpha)=\delta \alpha \gamma^{-1}$.

(ii) Definition of $M$. Let $\Gamma_{\Lambda}$ be set of matrices

$$
\gamma_{\lambda}:=\left(\begin{array}{cc}
\rho(\gamma) & \rho(\gamma) \lambda \\
0_{g \times 2} & \gamma
\end{array}\right) \in G l_{g+2}(\mathbb{R}), \quad \gamma \in \Gamma, \lambda \in \Lambda
$$

Then $\Gamma_{\Lambda} \simeq \Lambda \rtimes \Gamma$ and the sequence

$$
0 \longrightarrow \Lambda \longrightarrow \Gamma_{\Lambda} \longrightarrow \Gamma \longrightarrow 1
$$

given by $\lambda \mapsto i d_{\lambda}$ and $\gamma_{\lambda} \mapsto \gamma$ is exact. The projective action on $\mathbb{C}^{g} \times \mathfrak{H}_{1}$, i.e., where $\gamma_{\lambda} \in \Gamma_{\Lambda}$ acts by

$$
(z, \tau) \mapsto\left(\frac{\rho(\gamma)\left(z+\lambda\left(\begin{array}{l}
\tau \\
1
\end{array}\right)\right)}{c \tau+d}, \frac{a \tau+b}{c \tau+d}\right), \quad \gamma=\left(\begin{array}{ll}
a & b \\
c & d
\end{array}\right)
$$

is properly discontinously and free, since the action of $\Gamma$ and of each stabilizer group is. The quotient is a smooth complex manifold

$$
M=M_{\Gamma, \Lambda}:=\Gamma_{\Lambda} \backslash \mathbb{C}^{g} \times \mathfrak{H}_{1} .
$$

In the remainder of this subsection we prove:

5.6. Proposition. The manifold $M$ from above is a compact abelian group scheme over the Riemann surface $C_{\Gamma}:=\Gamma \backslash \mathfrak{H}_{1}$ and admits a projective structure.

$M$ has a projective structure by (1.4). By construction there is a natural holomorphic proper submersion $f: M \longrightarrow C_{\Gamma}$ with a section given by $[\tau] \mapsto[(0, \tau)]$. The fiber $M_{\tau}=f^{-1}([\tau])$ is isomorphic to $\mathbb{C}^{g}$ divided by the corresponding stabilizer subgroup of $\Gamma_{\Lambda}$. Since the action of $\Gamma$ on $\mathfrak{H}_{1}$ is free,

$$
M_{\tau} \simeq \mathbb{C}^{g} / \Lambda_{\tau},
$$


where $\Lambda_{\tau}$ is the image of $\Lambda$ under

$$
M_{g \times 2}(\mathbb{R}) \simeq W_{\mathbb{R}} \times U_{\mathbb{R}}^{*} \longrightarrow \mathbb{C}^{g}, \quad \alpha \mapsto \alpha_{\tau}:=\alpha\left(\begin{array}{c}
\tau \\
1
\end{array}\right) .
$$

(iii) Projectivity of $M_{\tau}$. (5.7) endows $M_{g \times 2}(\mathbb{R}) \simeq W_{\mathbb{R}} \otimes U_{\mathbb{R}}^{*}$ with the complex structure given by

$$
J_{\tau}=\frac{1}{\Im m \tau}\left(\begin{array}{cc}
-\Re e \tau & \tau \bar{\tau} \\
-1 & \Re e \tau
\end{array}\right) \in S l_{2}(\mathbb{R}), \quad \text { i.e., } \quad i \cdot \alpha_{\tau}=\left(\alpha J_{\tau}^{-1}\right)_{\tau} .
$$

If $\tau^{\prime}=\gamma(\tau)$ for some $\gamma \in S l_{2}(\mathbb{R})$, then $J_{\tau^{\prime}}=\gamma J_{\tau} \gamma^{-1}$. Hence $M_{\tau}$ is projective if $\left(M_{g \times 2}(\mathbb{R}) \simeq\right.$ $\left.W_{\mathbb{R}} \otimes U_{\mathbb{R}}^{*}, \Lambda, J_{\tau}, E\right)$ satisfies the Riemann conditions:

Recall that $M_{g \times 2}(\mathbb{R}) \simeq W_{\mathbb{R}} \otimes U_{\mathbb{R}}^{*}$ is a symplectic $O(S) \times S l_{2}(\mathbb{R})$-module. The complex structure is given by $\left(i d, J_{\tau}\right) \in O(S) \times S l_{2}(\mathbb{R})$. Therefore $E\left(\alpha J_{\tau}^{-1}, \alpha^{\prime} J_{\tau}^{-1}\right)=E\left(\alpha, \alpha^{\prime}\right)$, i.e., $E$ is compatible with the complex structure. Positivity for $\tau=i$ follows from $J_{i}=J_{2}$ and $E\left(\alpha, \alpha J_{2}^{-1}\right)=\operatorname{tr}\left(\alpha^{t} S \alpha\right)>0$ for $\alpha \neq 0$. Every $\tau \in \mathfrak{H}_{1}$ is of the form $\tau=\gamma(i)$ for some $\gamma \in S l_{2}(\mathbb{R})$. The invariance of $E$ and $J_{\tau}=\gamma J_{i} \gamma^{-1}$ shows $E\left(\alpha, \alpha^{\prime} J_{\tau}^{-1}\right)$ is a positive definite symmetric form for any $\tau \in \mathfrak{H}_{1}$. Therefore $E$ is a positive, integral $(1,1)$ form and $M_{\tau}$ is projective.

(iv) Isomorphic $M_{\tau}$ 's. If $\tau^{\prime}=\gamma(\tau)$ for some $\gamma \in \Gamma$, then $\left(M_{\tau}, E\right) \simeq\left(M_{\tau^{\prime}}, E\right)$, where $\varphi: M_{\tau} \longrightarrow M_{\tau^{\prime}}$, as a map $\mathbb{C}^{g} \longrightarrow \mathbb{C}^{g}$, is given by

$$
\frac{1}{c \tau+d} \rho(\gamma)
$$

Indeed,

$$
\Lambda_{\tau^{\prime}}=\Lambda\left(\begin{array}{c}
\gamma(\tau) \\
1
\end{array}\right)=\frac{1}{c \tau+d}(\Lambda \gamma)_{\tau}=\frac{\rho(\gamma)}{c \tau+d}\left(\rho\left(\gamma^{-1}\right) \Lambda \gamma\right)_{\tau}=\frac{\rho(\gamma)}{c \tau+d} \Lambda_{\tau}
$$

On the underlying real vector space $M_{g \times 2}(\mathbb{R}) \simeq W_{\mathbb{R}} \otimes U_{\mathbb{R}}^{*}, \varphi$ is given by $\alpha \mapsto \rho(\gamma) \alpha \gamma^{-1}$ showing that $\varphi$ preserves the polarization $E$.

The family of $M_{\tau}$ 's over $\mathfrak{H}_{1}$, i.e., the quotient $\mathbb{C}^{g} \times \mathfrak{H}_{1}$ divided by the action of the subgroup $\Lambda \subset \Gamma_{\Lambda}$ as in (5.5), is projective. We obtain $M$ by dividing out $\Gamma_{\Lambda} / \Lambda \simeq \Gamma$. Fiberwise this is nothing but (5.8) and the polarizations glue. Then $M$ is projective.

5.9. Remark. 1.) The quotient of $\Gamma \backslash \mathbb{C}^{g} \times \mathfrak{H}_{g}$, where $\gamma \in \Gamma$ acts as $\gamma_{0}$ (i.e., put $\lambda=0$ in (5.5) ) yields the fiberwise universal covering space $\simeq f_{*} T_{M / C_{\Gamma}}$ of $M \longrightarrow C_{\Gamma}$ (compare remarks 4.5 and 4.14). The total space also carries a projective structure. The local system $R^{1} f_{*} \mathbb{C}$ is given by the dual of $i d \otimes \rho$ (compare remark 4.14).

2.) Given a collection of data, we may replace $\rho$ by $\rho^{d}=\rho \oplus \cdots \oplus \rho$ and $W_{\mathbb{R}}$ by $W_{\mathbb{R}}^{d}$ for an arbitrary $d \in \mathbb{N}$. This again gives 1.)-3.) as in (5.1). It is clear from the construction that the new collection leads to $M_{\Gamma} \times_{C_{\Gamma}} M_{\Gamma} \times_{C_{\Gamma}} \cdots \times_{C_{\Gamma}} M_{\Gamma}$ with $d$ factors (compare (0.2), 3.).

5.10. From $A$ to $Z$. It remains to show how a quaternion algebra $A$ as in (0.2), 3.) leads to a collection of data 1.) - 3.) as in (5.1) (see also remark 5.9, 2.)). We first recall some results

(i) On central simple algebras. Let $A$ be a central simple algebra of finite dimension over a field $K$. It is called division if it is a skew field. It is called a quaternion algebra if $[A: K]=4$. A quaternion algebra is either division or split, i.e., $A \simeq M_{2}(K)$. Let $\operatorname{Br}(K)$ be the Brauer group of $K$. The order $e(A)$ of $[A] \in B r(K)$ is finite and is called the exponent of $A$. A theorem of Wedderburn says $A \simeq M_{r}(D)$, where $D / K$ is a division algebra. The $K$-dimension $[D: K]=s(D)^{2}$ for some $s(D)=s(A) \in \mathbb{N}$ is called (Schur-) index of $A$. One has $e(A) \mid s(A)$ and if $K$ is a local or global field, then even $e(A)=s(A)$. 
(ii) The construction. The corestriction $\operatorname{Cor}_{F / \mathbb{Q}}(A)$ is a $4^{d}$ dimensional central simple $\mathbb{Q}$-algebra. The corestriction induces a map of Brauer groups

$$
\operatorname{Br}(F) \longrightarrow \operatorname{Br}(\mathbb{Q}) \text {. }
$$

5.12. Lemma. For $A$ as in (5.2)

$$
\operatorname{Cor}_{F / \mathbb{Q}}(A) \simeq M_{2^{d}}(\mathbb{Q}) \text { or } \operatorname{Cor}_{F / \mathbb{Q}}(A) \simeq M_{2^{d-1}}(B),
$$

where $B$ is a division quaternion algebra over $\mathbb{Q}$ which is indefinite (i.e., $B \otimes \mathbb{R} \simeq M_{2}(\mathbb{R})$ ) iff $d$ is even, definite (i.e., $B \otimes \mathbb{R} \simeq \mathbb{H}$ ) iff $d$ is odd.

Proof. The order of $[A]$ in $\operatorname{Br}(F)$ is $e(A)=s(A)=2$. Consider (5.11). We find $e\left(\operatorname{Cor}_{F / \mathbb{Q}}(A)\right)=1$ or 2 , implying (5.13). From $A \otimes_{\mathbb{Q}} \mathbb{R} \simeq M_{2}(\mathbb{R}) \oplus \mathbb{H}^{\oplus d-1}$ using $\mathbb{H} \otimes_{\mathbb{R}} \mathbb{H} \simeq$ $M_{4}(\mathbb{R})$ we obtain

$$
\operatorname{Cor}_{F / \mathbb{Q}}(A) \otimes \mathbb{Q} \mathbb{R} \simeq M_{2}(\mathbb{R}) \otimes_{\mathbb{R}} \mathbb{H}^{\otimes_{\mathbb{R}} d-1} \simeq \begin{cases}M_{2^{d}}(\mathbb{R}), & d \text { odd } \\ M_{2^{d-1}}(\mathbb{H}), & d \text { even }\end{cases}
$$

The statement on $B \otimes \mathbb{R}$ now follows from combining (5.14) and (5.13).

We refer to the first case in (5.13) as " $B$ splits", because here $\operatorname{Cor}_{F / \mathbb{Q}}(A) \simeq M_{2^{d-1}}(B)$ for $B=M_{2}(\mathbb{Q})$. Consider the following $\mathbb{Q}$-vector spaces

$$
V_{\mathbb{Q}}=\mathbb{Q}^{2^{d}} \text {, in the case } B \text { split, } V_{\mathbb{Q}}=B^{2^{d-1}} \text {, in the case } B \text { non-split. }
$$

The $\mathbb{Q}$-dimension is $2^{d}$ and $2^{d+1}$, respectively. The elements of $V_{\mathbb{Q}}$ are considered as vertical vectors and $V_{\mathbb{Q}}$ as a left $\operatorname{Cor}_{F / \mathbb{Q}}(A)$-module.

Over the reals we have by (5.14) $\operatorname{Cor}_{F / \mathbb{Q}}(A) \otimes \mathbb{Q} \mathbb{R} \simeq M_{2}(\mathbb{R}) \otimes_{\mathbb{R}} \mathbb{H}^{\otimes_{\mathbb{R}}} d-1 \simeq$

$$
\simeq \begin{cases}M_{2}(\mathbb{R}) \otimes M_{2^{d-1}}(\mathbb{R}), & B \text { indefinite } / d \text { odd } \\ M_{2}(\mathbb{R}) \otimes M_{2^{d-2}}(\mathbb{H}), & B \text { definite } / d \text { even }\end{cases}
$$

Consider the following left $\operatorname{Cor}_{F / \mathbb{Q}}(A) \otimes_{\mathbb{Q}} \mathbb{R}$-modules: $\mathbb{R}^{2} \otimes \mathbb{R}^{2^{d-1}}$ in the case $B$ split, $\mathbb{R}^{2} \otimes M_{2^{d-1}, 2}(\mathbb{R})$ in the case $B$ non-split indefinite and finally $\mathbb{R}^{2} \otimes \mathbb{H}^{2^{d-2}}$ in the case $B$ definite. In all three cases, denote the first factor by $U_{\mathbb{R}}=\mathbb{R}^{2}$, the second by $W_{\mathbb{R}}$. Then $U_{\mathbb{R}} \otimes W_{\mathbb{R}} \simeq V_{\mathbb{Q}} \otimes \mathbb{R}$ as left $\operatorname{Cor}_{F / \mathbb{Q}}(A) \otimes \mathbb{Q} \mathbb{R}$ modules, i.e., the action of $\operatorname{Cor}_{F / \mathbb{Q}}(A) \otimes \mathbb{Q} \mathbb{R}$ on $U_{\mathbb{R}} \otimes W_{\mathbb{R}}$ is a real form of the action of $\operatorname{Cor}_{F / \mathbb{Q}}(A)$ on $V_{\mathbb{Q}}$.

The corestriction comes with a map

$$
N m: A^{\times} \longrightarrow \operatorname{Cor}_{F / \mathbb{Q}}(A)^{\times} .
$$

Let $x \mapsto x^{\prime}$ be the canonical involution of $A$ and

$$
G=\left\{x \in A \mid x x^{\prime}=1\right\} .
$$

Then $G$ is an algebraic group over $\mathbb{Q}$. Via $N m, G(\mathbb{Q})$ acts on $V_{\mathbb{Q}}$. Let $\Lambda \subset V_{\mathbb{Q}}$ be some complete lattice, $\Gamma \subset G(\mathbb{Q})$ be some torsion free arithmetic subgroup fixing $\Lambda$ via $N \mathrm{~m}$.

Consider the action of $G$ on $V_{\mathbb{Q}}$ over $\mathbb{R}$ : The elements of norm 1 in $M_{2}(\mathbb{R})$ and $\mathbb{H}$ form the groups $S l_{2}(\mathbb{R})$ and $S U(2)$, respectively. From $A \otimes_{\mathbb{Q}} \mathbb{R} \simeq M_{2}(\mathbb{R}) \oplus \mathbb{H}^{\oplus d-1}$ we infer

$$
G(\mathbb{R})=S l_{2}(\mathbb{R}) \times \underbrace{S U(2) \times \cdots \times S U(2)}_{d-1} .
$$

The isomorphism $\mathbb{H} \otimes_{\mathbb{R}} \mathbb{H} \simeq M_{4}(\mathbb{R})$ induces $S U(2) \times S U(2) \longrightarrow S O(4)$. Then $N m$ factors over

$$
G(\mathbb{R}) \longrightarrow \begin{cases}S l_{2}(\mathbb{R}) \times S O\left(2^{d-1}\right), & B \text { indefinit } / d \text { odd } \\ S l_{2}(\mathbb{R}) \times S U\left(2^{d-1}\right), & B \text { definit } / d \text { even }\end{cases}
$$


Projection onto the first factor embeds $\Gamma$ into $S l_{2}(\mathbb{R})$ and gives $U_{\mathbb{R}}$ from above the usual symplectic structure as a $\Gamma$-module. Projection onto the second factor gives $W_{\mathbb{R}}$ the structure of an orthogonal $\Gamma$-module (in the case $W_{\mathbb{R}}=\mathbb{H}^{2^{d-2}}$ take the real part of the unitary form). From (5.16) we see that $\Gamma$ fixes a symplectic form $E$ obtained by tensorising the standard symplectic form on $U_{\mathbb{R}}$ and some orthogonal form on $W_{\mathbb{R}}$ such that $E$ only takes integral values on $\Lambda$. This gives 1.) - 3.) as in (5.1).

5.17. Remark. The group $G$ can be identified with the special Mumford Tate group of the constructed family of abelian varieties. The $\operatorname{ring} E n d_{\mathbb{Q}}\left(Z_{\tau}\right)$ for $\tau$ general is isomorphic to the group of endomorphisms of $V$ commuting with the action of $G$.

In the case $B$ non split, $V_{\mathbb{Q}}$ becomes a $B$-module via $\beta v:=v \beta^{\prime}$ and this action clearly commutes. This gives an embedding $B \hookrightarrow \operatorname{End}_{\mathbb{Q}}\left(Z_{\tau}\right)$. By (ViZu04, Lemma 6.9., here $B$ is not explicitely mentioned) we find $\operatorname{End}_{\mathbb{Q}}\left(Z_{\tau}\right) \simeq B$ in the case $B$ non-split and $\operatorname{End}_{\mathbb{Q}}\left(Z_{\tau}\right) \simeq \mathbb{Q}$ in the case $B$ split.

5.18. Example.(False elliptic Curves) (Sh59]) We conclude by describing how the families of false elliptic curves already explained in (0.3) fit into the general picture in (5.1).

In the above setting it is the case $d=1, F=\mathbb{Q}, A=B$ indefinit. Denote quaternion conjugation in $B$ by $^{\prime}$. Choose some pure quaternion $y$ (i.e., $y=-y^{\prime}$ ) such that $b:=y^{2}<0$. There exists $x \in B$ such that $x y=-y x$ and $a:=x^{2}>0$. Then $B$ is generated by $x$ and $y$ as an algebra over $\mathbb{Q}$ and

$$
x \mapsto\left(\begin{array}{cc}
\sqrt{a} & 0 \\
0 & -\sqrt{a}
\end{array}\right), \quad y \mapsto\left(\begin{array}{cc}
0 & b \\
1 & 0
\end{array}\right)
$$

gives an emedding $B \hookrightarrow M_{2 \times 2}(\mathbb{R})$ and a basis of $U_{\mathbb{R}}=\mathbb{R}^{2}$. If we identify $B$ with its image in $M_{2 \times 2}(\mathbb{R})$, s.t. reduced norm and trace are given by usual matrix determinant and trace, respectively, then we are in situation at the beginning (0.3).

Here $V_{\mathbb{Q}}=B$ and we have to choose a complete lattice $\Lambda$ in $B$, for example a maximal order. For $\Gamma$ we have to choose an arithmetic torsion free subgroup of the norm one unit subgroup $\Lambda_{1}^{\times} \subset \mathrm{Sl}_{2}(\mathbb{R})$. For $\rho: \Gamma \longrightarrow O\left(W_{\mathbb{R}}=\mathbb{R}^{2}, 2\right)$ we have to choose the trivial representation, as $d=1$. This gives a collection of data 1.)-3.) as in (5.1). Indeed, identify $W_{\mathbb{R}} \otimes U_{\mathbb{R}}^{*}=M_{2}(\mathbb{R})$, where $M_{2}(\mathbb{R})$ is the space containing $B$. $\Gamma$ acts on $M_{2}(\mathbb{R})$ via $\alpha \mapsto \rho(\gamma) \alpha \gamma^{-1}=\alpha \gamma^{-1}$, fixing the lattice $\Lambda$. In (0.3) and in (5.1) the same group $\Gamma_{\Lambda} \subset \mathrm{Sl}_{4}(\mathbb{R})$ is defined leading to the same quotient $\Gamma_{\Lambda} \backslash \mathbb{C}^{2} \times \mathfrak{H}_{1}$.

For the positive symmetric form on $W_{\mathbb{R}}$ we choose $S_{1}:=J_{2} y$. Then $E_{1}(\alpha, \beta)=$ $\operatorname{tr}\left(\alpha^{t} S_{1} \beta J_{2}\right)$ is a $\Gamma$-stable symplectic form on $M_{2}(\mathbb{R})$. The extension of quaternion conjugation from $B$ to $M_{2}(\mathbb{R})$ is given by $\alpha \mapsto J_{2}^{-1} \alpha^{t} J_{2}$. Then $($ recall $\operatorname{tr}(\alpha \beta)=\operatorname{tr}(\beta \alpha)$ in $\left.M_{2}(\mathbb{R})\right)$

$$
\begin{gathered}
E_{1}(\alpha, \beta)=\operatorname{tr}\left(\alpha^{t} S \beta J_{2}\right)=\operatorname{tr}\left(-J_{2} \beta^{t} S \alpha\right)=\operatorname{tr}\left(-J_{2} y \alpha J_{2} \beta^{t}\right)= \\
=\operatorname{tr}\left(y \alpha J_{2}^{-1} \beta^{t} J_{2}\right)=\operatorname{tr}\left(y \alpha \beta^{\prime}\right) .
\end{gathered}
$$

The last description shows that $E_{1}$ only takes rational values on $B$. Then some multiple of $E_{1}$ only takes integral values on $\Lambda$ which gives the desired form $E$ (Shimura's form from Sh59]).

\section{REFERENCES}

[Au76] T. Aubin: Equations du type Monge-Ampère sur les variété Kählériennes compactes. C. R. Acad. Scie. Paris 283 (1976), 119-121

[At57] M.F. Atiyah: Complex analytic conections in fibre bundles. AMS Transactions 85 (1957), 181-207

[Be83] A. Beauville: Variété Kähleriennes dont la premiere classe de Chern est nulle. J. Diff. Geom. 18 (1983), 755-782 
[BiLa04] Ch. Birkenhake, Lange: Complex Abelian Varieties. Grundlehren der Math. Wiss. 302, Springer (2004)

[Bo74] F. Bogomolov: On the decomposition of Kähler manifolds with trivial canonical class. Math. USSR Sbornick 22 (1974), 580-583

[BrHaNo32] R. Brauer, H. Hasse, E. Noether: Beweis eines Hauptsatzes in der Theorie der Algebren. J. reine angw. Math. 167 (1932), 399-404

[ChOg75] B.Y. Chen, K. Ogiue: Some characterization of complex space forms in terms of Chern classes. Quart. Journ. Math. Oxford 26 (1975), 459-464

[Fa83] G. Faltings: Arakelov's theorem for abelian varieties, Inv. Math. 73 (1983), 337-347

[Gu78] R. Gunning: On uniformization of complex manifolds: The role of connections. Princeton University Press (1978)

[Ha77] R. Hartshorne: Algebraic Geometry. Springer GTM 52 (1977)

[Hi81] N.J. Hitchin: Kaehlerian twistor spaces. Proc. Lond. Math. Soc., III, Ser. 43 (1981), 133-150

[HwMo97] J.M. Hwang, N. Mok: Uniruled projective manifolds with irreducible reductive $G$-structures. J. reine angew. Math. 490 (1997), 55-64

[HwMo98] J.M. Hwang, N. Mok: Rigidity of irreducible Hermitian symmetric spaces of the compact type under Kähler deformation. Inv. Math. 131, 393-418 (1998)

[JaRa04a] P. Jahnke, I. Radloff: Threefolds with holomorphic normal projective connections. Math. Ann. 329 (2004), 379-400

[JaRa04b] P. Jahnke, I. Radloff: Splitting jet sequences. Math. Res. Letters 11 (2004), 345-354

[JaRa05] P. Jahnke, I. Radloff: Projective threefolds with holomorphic conformal structure. Int. J. Math. 16, No.6 (2005), 595-607

[JoZu02] J. Jost, K. Zuo: Arakelov type inequalities for Hodge bundles over algebraic varieties, Part 1: Hodge bundles over algebraic curves. J. Alg. Geom. 11 (2002), 535-546

[Ka85] Y. Kawamata: Minimal models and the Kodaira dimension of algebraic fiber spaces. J. reine angew. Math. 363 (1985), 1-46.

[Ka91] Y. Kawamata: On the length of an extremal rational curve. Inv. Math. 105 (1991), 609-611

[Ko93] J. Kollár: Shafarevich maps and plurigenera of algebraic varieties, Inv. Math. 113 (1993), 176-215

[KoOc80] S. Kobayashi, T. Ochiai: Holomorphic projective structures on compact complex surfaces I, II. Math. Ann. 249 (1980), 75-94; 255 (1981), 519-521

[KoOc81] S. Kobayashi, T. Ochiai: Holomorphic structures modeled after compact Hermitian symmetric spaces. Manifolds and Lie groups, Pap. in Honor of Y. Matsushima, Prog. Math. 14 (1981), 207-222

[KoMo98] J. Kollár, S. Mori: Birational Geometry of Algebraic Varieties. Cambridge tracts in mathematics 134 (1998)

[KoWu83] S. Kobayashi, H. Wu: Complex Differential Geometry. DMV Seminar 3, Birkhäuser (1983)

[Lai11] C.J. Lai: Varieties fibered by good minimal models. Math Ann. 350 (2011), 533-547

[MoMo96] R. Molzon, K. Mortensen: The Schwarzian derivative for maps between manifolds with complex projective connections. Tr. AMS 348, (1996), 3015-3036

[MiPe97] Y. Miyaoka, T. Peternell: Geometry of higher dimensional algebraic varieties, DMV Seminar 26, Birkhäuser (1997)

[Mori79] S. Mori: Projective manifolds with ample tangent bundles. Ann. Math. 110 (1979), 593-696

[Mori87] S. Mori: Classification of higher dimensional varieties, Proc. Symp. Pure Math., 46, 269-331 (1987)

[Mu66] D. Mumford: A note of Shimura's paper: Discontinuous groups and Abelian varietes. Math. Ann. 181 (1969), 345-351

[Na99a] N. Nakayama: Projective algebraic varieties whose universal covering spaces are biholomorphic to $C^{n}$. J. Math. Soc. Japan 51 (1999), 643-654

[Na99b] N. Nakayama: Compact Kähler manifolds whose universal covering spaces are biholomorphic to $C^{n}$. RIMS Preprint 1230 (1999)

[Sa80] I. Satake: Algebraic structures of symmetric domains. Kano Memorial Lectures 4. Publ. Math. Soc. Japan, 14. Tokyo: Iwanami Shoten (1980)

[Sa97] I. Satake: Equivariant holomorphic embeddings of symmetric domains and applications. RIMS Kokyuroku 1002 (1997), 19-31

[Sh59] G. Shimura: On the theory of automorphic functions. Ann. of Math. 70 (1959), 101-144

[Si10] Y.T. Siu: Abundance conjecture. In Geometry and analysis, 271-317. Adv. Lectures in Math. Boston: Int. Press. (2010) 
[Ta75] K. Takeuchi: A characterization of arithmetic Fuchsian groups. J. Math. Soc. Japan 27 (1975), 591-596

[vGe08] B. van Geemen: Real multiplication on K3 surfaces and Kuga Satake varieties, Michigan Math. J. 56 (2008), 375-399

[KoVe03] D. Kohel, H. Verrill: Fundamental domains of Shimura curves. J. Théor. Nombres Bordx. 15 (2003), 205-222

[ViZu04] E. Viehweg, K. Zuo: A characterization of certain Shimura curves in the moduli stack of abelian varieties. J. Diff. Geom 66 (2004), 233-287

[ViZu07] E. Viehweg, K. Zuo: Arakelov inequalities and the uniformization of certain rigid Shimura varieties. J. Diff. Geom 77 (2007), 291-352

[Ya78] S.T. Yau: On the Ricci curvature of a compact Kähler manifold and the complex MongeAmpére equation, I. Comm. Pure Appl. Math. 31 (1978), 339-411

[Zu96] K. Zuo: Kodaira dimension and Chern hyperbolicity of the Shafarevich maps for representations of $\pi_{1}$ of compact Kähler manifolds, J. reine angew. Math. 472 (1996), 139-156

Priska Jahnke - Fakultät T1 - Hochschule Heilbronn - MaX-Planck-Strasse 39 - D-74081 Heilbronn, Germany

E-mail address: priska.jahnke@hs-heilbronn.de

Ivo Radloff - Mathematisches Institut - Universität Tübingen - Auf Der Morgenstelle 10 - D-72076 Tübingen, Germany

E-mail address: ivo.radloff@uni-tuebingen.de 